\title{
The Classification and Characteristics of Soil Surrounding Lake Ecosystem as a Basic Consideration in Agrotourism Development in Linow Lake, North Sulawesi.
}

\author{
Diane D. Pioh ${ }^{1}$, M. Luthfi Rayes ${ }^{2}$, Boby Polii ${ }^{3}$, Luchman Hakim ${ }^{4}$ \\ ${ }^{1}$ Graduate School of Agriculture, Faculty of Agriculture University of Brawijaya, Malang and Faculty of \\ Agriculture, University of Sam Ratulangi, Manado, North Sulawesi, Indonesia \\ ${ }^{2}$ Faculty of Agriculture, University of Brawijaya, Malang, Indonesia \\ ${ }^{3}$ Faculty of Agriculture, University of Sam Ratulangi, Manado, North Sulawesi, Indonesia \\ ${ }^{4}$ Faculty of Mathematics and Natural Sciences, University of Brawijaya, Malang, Indonesia
}

\begin{abstract}
The objective of the research is to identify and classify soil in an area surrounding Linow Lake, North Sulawesi for agrotourism development purposes. Result of the study confirm that lands surrounding Linow Lake can be classified into nine lands unit. The landform of these units dominated by hilly volcanic and soils were varies namely Aquic eutrudept, Humic dystrudept, Typic dystrudept, humic hapludult, Typica sulfudept and sulfic endoaquept. Soils in area surrounding Linow Lake can be classified into two orders namely Inceptisols and Ultisols. Inceptisols is characterized by cambic horizon as shown on soil profile 1,2,6 and 7. Ultisols is characterized by the existence of argillic B horizon, as shown on soil profile 4,5 and 8. The possibility for agrotourism development lies on the opportunities to plant some fruit trees, i.e. Rambutan, Lansat, Avocado, Durian, Guava, Papaya and Banana in policulture systems, or technically called agroforestry. The planting arragement and management should be meets the landcapability and suitability.
\end{abstract}

Keywords: environmental management, soil classification, lake ecosystem, tourism development

\section{Introduction}

Tourism is fast growing industry in North Sulawesi Indonesia. Scholars point out that natural richness becomes the key attraction for tourism development in North Sulawesi. North Sulawesi endowed by spectacular terrestrial marine biodiversity. Many diving spot has been visited by tourist, including Bunaken, Siladen, Mantehage, Manado Tua, Nain, Likupang, Talise, Bangka and Lembeh. These areas nowadays become the ultimate marine tourism destination in the world. Besides marine based attraction, there are active volcanic, lakes, plantation, hot spring and national parks available for tourism. Principally, a growing number of tourists in such area are exploring natural beauty and seeking new experiences [1] [2].

Rapid development and increasing concern in nature based tourism has been lead lakes ecosystems used as tourism attractions. Globally, there are numerous spectacular lakes which are visited by thousand and millions tourist. In Indonesia, Toba Lake (North Sumatra), Buyan Lake (Bali) and Kelimutu Lake (Flores) are the example of lake-based tourism destination. In Sulawesi, Tondano Lake has been knows as one of the ultimate lake-based tourism attraction [3]. The problems of lake-based tourism implementation often related to the decrease of lakes quality, both its aquatic and surrounding terrestrial ecosystems. The water are often facings erosion problem, eutrophication and invasion of exotic plants species (i.e. Eichhornia crassipes, Salvinia sp., etc). Lands surrounding lakes often miss managed properly and led to the rapid lakes degradations [4] [5].

Lands and ecosystems management surrounding lakes has been viewed as an integral part towards sustainable lake-based tourism destinations. There is numerous evidences that unsustainable agricultural practices lead to lakes eutrophications. Intensive agriculture in riparian areas causes decrease of riparian function as lakes buffer. In the perspectives of physical development, land surrounding lakes is interesting sites to build numerous recreation facility and tourism infrastructure. Such land is the potential space for agriculture sites that can be integrated into tourism attractions system. It is particularly important to increase sites attractiveness and support lake-based tourism industry. In such scenarios development, determining lands viability and suitability to enhance sustainable lake-based tourism area becomes important [6] [7].

Agricultural area in adjacent to the Linow Lake in Tomohon, North Sulawesi, potentially can be developed in harmony with lake-based tourism. The interaction of lake based tourism and agrotourism opens new opportunities for destination development. The functions of terrestrial area adjacent to the lake can be planned and diversified into agrotourism area. In such a case, lands evaluation becomes the crucial keys in spatial planning and development. The data and information of land suitability for sustainable agriculture which are related to tourism development is crucial. In agriculture development, land suitability closely related to soil. Soil is source of water and plant's nutrients. Soil are complex and it is characterized by soil texture, structure, 
and its chemical properties. Soil character especially important in lands management, especially in land rehabilitation and ecosystem restoration programs [8] [9] [10]. The objective of the research is to identify and classify soil in an area surrounding Linow Lake, North Sulawesi for agrotourism development purposes.

\section{Study area}

\section{Methodology}

Linow Lake located in the hearth of North Sulawesi province. Administratively, Linow Lake belongs to Tomohon City, with the city area is about $147.21 \mathrm{Km}^{2}$. Tomohon located at the highland area (ca. $700-900 \mathrm{~m}$ asl) and the city is surrounded by active volcanoes namely Mt. Masarang (560 m), Mt. Lokon (1.689 m) and Mt. Mahawu $(1.311 \mathrm{~m})$. Tomohon recently grows as tourism area with numerous offered natural and cultural attractions. It is encompasses hiking in Mt. Lokon and Mt. Mahawu, refreshing in Linow Lake, TumimperasPinaras waterfall, Tapahan Telu Tinor Waterfall, Temboan Hill, Lahendong forest park. There are also numerous culinary, handicraft and cultural events which are represent the characteristics of Tomohon City. Tomohon has been known as flower producers. Regularly local government performs Tomohon Flower Festival as cultural tourism events [11].

Linow Lake is one of the natural tourism attractions in Tomohon City. Geologically, Linow Lake is formed as a result of volcanic activity in central part of North Sulawesi region. There are some sulfur bubble in some point in lakes, indicates that Linow Lake is located in the very active volcanic areas. The water of lakes become green and sometimes blues as an impact of sulfur in fresh lakes water. Recently, the infrastructure and access to lakes has been improved to increase tourist visitation and satisfaction. The threats to the lake however, come from potential miss management of land surrounding lake. The lands surrounding lake recently dominated by barren land and agriculture lands.

The study area is located between $0117^{\prime} 50-01^{\circ} 18^{\prime} 51^{\prime \prime} \mathrm{N}$ and $124^{\circ} 49^{\prime} 00^{\prime \prime}-124^{\circ} 50^{\prime} 30^{\prime \prime} \mathrm{E}$ and ranged in elevation from 780 to $900 \mathrm{~m}$ asl. The underlying parent material within the study area consists primarily of volcanic materials. The study area is predominantly used for mix garden and dry land Agriculture.

\section{Methods}

Field work was conducted in 2013 following the sequential stages, namely collecting secondary data (i.e. topographic map, land use map, climatic data), construction map unit of the area, field work and laboratory analysis and land suitability assessment for different crops. Topographic and land use map was collected from National Coordinating Agency for Surveys and Mapping (BAKOSURTANAL), Jakarta. These map attributes are analyzed and classified using ArcGIS 10.2 software.

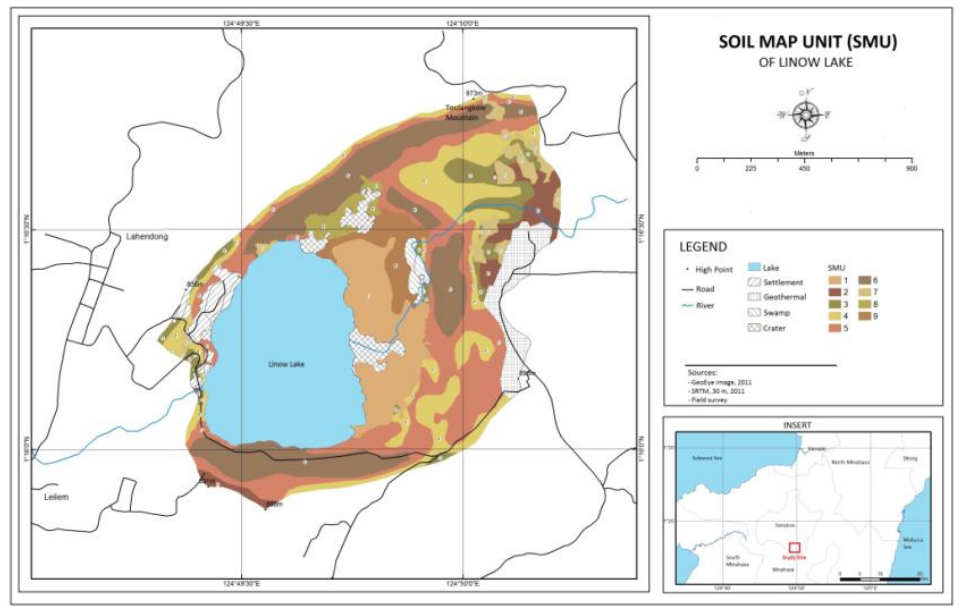

Fig. 1. Location of the study area in Tomohon City, North Sulawesi

\section{Soil Survey and Land Evaluation}

There were nine land units found in this study area (Fig 1). Land unit is derived by overlaying topographic map, slope map and land use map. Field observations have been done according to Rayes (2006) [12]. Using the land unit as a spatial data source and orientation, 35 minipit and augering have been done to get information about type of soil found on each land unit. Based on those data seven soil profiles formed on selective land units were observed and sampled. A total of thirty eight soil samples were collected from each horizon of seven soil profiles for laboratory analysis. Soils were classified according to Soil Taxonomy [13] [14]. 


\section{Soil analysis}

The physical and chemical soil analysis has been done following standard procedures. All soil samples were air dried, crushed and screened using a $2 \mathrm{~mm}$ sieve. Soil texture was determined by the hydrometer method [15], and bulk density was determined from undisturbed samples. Organic matter was determined by the Walkley-Black wet digestion method. Nitrogen in soil was determined by the Kjeldahl method [16] and soil pH was determined according to the standard methods of soil analysis. Cation exchange capacities (CEC) were measured by using the $1 \mathrm{~N} \mathrm{NH}_{4} \mathrm{OAC}$ (pH 7) method (Soil Survey Laboratory, 1992). Exch-Al and exch-H were determined using van Reeuwijk (2002) methods [17]. Soils were classified according to Soil Survey Staff classification [13].

\section{Land suitability assessment for agrotourism development}

The land suitability classification was made following the methods of FAO (FAO, 1976). Automated Land Evaluation System (ALES) [18] was employed for the land evaluation. Steps to perform land evaluation of the study area were done systematically, namely: 1) selecting fruit trees or commodities to be developed in the study area, 2) deciding on land use requirements and factor ratings based on Djaenudin (1997) criteria [19], 3) preparing the relevant land qualities and land characteristic for each land map unit and finally, and 4) matching land use requirements with land qualities using the Automated Land Evaluation Systems (ALES).

\section{Climatic data}

\section{Results and Discussions}

Rainfall data recorded since 2001 to 2010 (10 years) from weather station of Kayuwatu Manado, North Sulawesi is shown on Fig. 3 [11] [20]. According to Köppen climate classification, the study area has features a tropical rainforest climate (Af) [21]. The annual precipitation of the area was an average of $2829.4 \mathrm{~mm}$. The study area has a noticeably rainier season that start from November through June and a drier season that covers the remaining four months. However, this area does not have a true dry season because the lowest monthly precipitation was $57.4 \mathrm{~mm}$ (on August). The average temperatures of the area under study remain constant throughout the year, with an average of $22.5^{\circ} \mathrm{C}$. The highest temperatures is on May $\left(23^{\circ} \mathrm{C}\right)$ and the lowest temperature being on August $\left(22.2^{\circ} \mathrm{C}\right)$. The soil temperature regime of the area belongs to Isohyperthermic and the soil moisture regime is classified as udic (Fig.2).

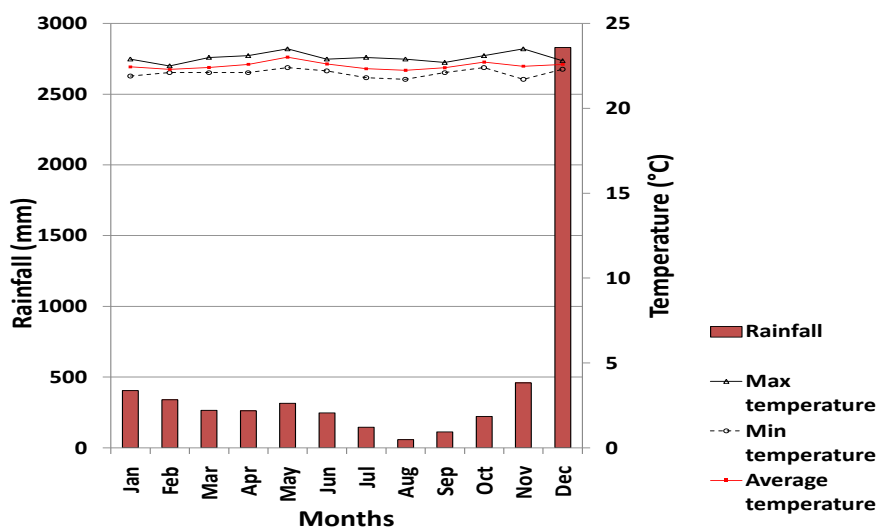

Fig. 2. Mean monthly rainfall, minimum, maximum and average temperature of study area

The tropical rain forest ultimately supports numerous plants species to grows, ranging from orchids to giant trees. In such ecosystems, numerous tropical fruits grows wild and provides continuous economic benefits for people in tropical forest [22].

\section{Soil morphology, characteristics and classification}

The soils in the study area were developed from volcanic activity of Linow Lake. Land unit 1 and 2 belongs to lacustrine of the lake and most of the time is inundated with water. The morphology properties of 7 soil profiles were summaries on Table 1.

Profile 1 (P1) developed on lower slope area of mix garden land use, that get supply of top soil eroded from upper part, therefore the A horizon have a dark color. Clay loam was the dominant texture throughout the Profile 1. The epipedon was umbric due to the dark color (moist and dry) of A and Bw1 horizons but the base saturation was lower than $50 \%$ (Table 2). The diagnostic sub horizon was cambic $(11-100 \mathrm{~cm})$. This profile was classified as Humic Dystrudept [13]. 
Profile 5 (P5) located on land unit 4 (15-25\% slope) have an ochric epipedon due to lighter color and has a cambic horizon (11-100 cm depth). Base saturation on cambic horizons were around $28 \%$ in average (Table 2) therefore the soil is classified as Typic Dystrudepts.

Profile 4 (P4), P5 and P9 have the same morphology properties, but they were located on different land unit (Table 1). Profile 4 (P4) located on land unit 5 (25-40\% slopes), profile 5 (P5) located on land unit 6 (>40\% slopes) and Profile 9 (P9) located on land unit 7 (15-25\% slopes). They were have the same morphology properties that quite different from those two previous profiles. The morphology of P4, P5 and P9 were defined as A-AB-Bt1-Bt2-Bt3-Bt4-Bt5 (Table 1). The top soils had a clay loam texture while the whole B horizon had a clay loam to clay texture. The increases of clay particles on those B horizon is attributable to clays eluviations down to the lower horizons and accumulating as a thin membrane the so called a cutan (clay skin) on the surfaces of sub-angular blocky structures of the B horizon. The main subsurface diagnostic horizon in P4 was an argillic horizon, while the surface diagnostic horizon was an umbric epipedon. The epipedon fulfill the requirement of a mollic epipedon but the base saturation was less than $50 \%$ (Table 2). Scholar point out that an argillic horizon must contain a minimum clay increase relative to the elluvial horizon or an underlying horizon, and show evidence of clay movement [23]. This process of soil formation was called lessivage or argilluviation. This profile was classified as Humic Hapludults, due to lower base saturation of the argillic horizon.

Profile 2 (P2) located on Land Unit 8 (25-40\% slopes) was strongly affected by sulphur materials from the volcanic lake. On this land unit sulphur outcrops were observed. The epipedon was ochric due to low content of C-organic. Within the $50 \mathrm{~cm}$ of the soil surface thet soil has a sulfuric horizons indicated by an elemental sulfur which form sulfuric acid. The horizon was cambic, therefore the soil classified as Typic Sulfudepts.

Profile 8 (P8) located on lower slopes closed to the lake. The land were formerly use for growing rice. This time no longer use as rice field due to the $\mathrm{pH}$ (less than 4, Table 2), that make the land being not suitable for rice crops. Soil was saturated with water in all layers from the surface to the bottom of the profile. In the upper part of the profile several colors (mottling or redoximorphic features) recorded due to loss (depletion) or gain (concentration) of pigment compared to the matrix color which is formed by oxidation/reduction of iron and/or manganese coupled with their removal and translocation [24]. The gray color of the subsoil is an evidence of seasonal rise in water table or water logging. Based on that the soil moisture regime, this land unit was calssified as Aquic. Epipedon were an ochric due to the lighter color to be a mollic or an umbric epipedon. A Cambic horizon was observed on the profile that is why the soil be classified as Inceptisols. Due to the fact that the soil is saturated with water in all layers from the surface to the bottom of the profile the soil has aquic condition as episaturation [13]. On horizons Bwg1 and Bwg2 has all the characteristic of a sulfuric horizons except the pH 3.7-4.0 and did not have sulfur bearing minerals; the soil was classified as Sulfic Endoaquepts. Following Soil Survey Staff (2010) [13], the distribution of soil in subgroup category is shown on Fig.3.

Table 1. Some Morphological Characteristics of Soil Profiles

\begin{tabular}{|c|c|c|c|c|c|c|c|}
\hline $\begin{array}{c}\text { Profiles/LU } \\
\text { Horizon }\end{array}$ & Depth $(\mathrm{cm})$ & $\begin{array}{l}\text { Colours } \\
\text { (moist) }\end{array}$ & $\begin{array}{l}\text { Colours } \\
\text { (dry) }\end{array}$ & Texture & Structure & $\begin{array}{l}\text { Consis- } \\
\text { tence }\end{array}$ & Boundary \\
\hline 1 & 3 & 4 & 5 & 6 & 7 & 8 & 11 \\
\hline \multicolumn{8}{|c|}{ Profiles 1/3 Humic Dystrudepts $\quad 8-15 \%$ mix garden } \\
\hline A & $0-11$ & $10 \mathrm{YR} 3 / 3$ & $10 \mathrm{YR} 4 / 5$ & Lpli & $\mathrm{gb}, \mathrm{hl}, \mathrm{sd}$ & $\mathrm{g}$ & ba,r \\
\hline Bw1 & $11-29$ & $10 \mathrm{YR} 3 / 3$ & $10 \mathrm{YR} 4 / 5$ & Lpli & $\mathrm{gb}, \mathrm{hl}, \mathrm{sd}$ & $\mathrm{g}$ & ba,r \\
\hline Bw2 & $29-58$ & $10 \mathrm{YR} 4 / 3$ & - & Lpli & $\mathrm{gb}, \mathrm{hl}, \mathrm{sd}$ & $\mathrm{g}$ & bu,r \\
\hline Bw3 & $58-100$ & $10 \mathrm{YR} 4 / 3-4 / 4$ & - & lpli & $\mathrm{gb}, \mathrm{hl}, \mathrm{sd}$ & $\mathrm{g}$ & bu,r \\
\hline $\mathrm{BC}$ & $100-150$ & $10 \mathrm{YR} 4 / 4$ & - & lpli & $\mathrm{gb}, \mathrm{hl}, \mathrm{sd}$ & $\mathrm{g}$ & bu,r \\
\hline \multicolumn{8}{|c|}{ Profiles 6/4 Typic Dystrudepts, $15-25 \%$ mix garden } \\
\hline A & $0-11 / 13$ & $10 \mathrm{YR} 3 / 4$ & - & lpli & $\mathrm{gb}, \mathrm{hl}, \mathrm{sd}$ & $\mathrm{sg}$ & ba,bo \\
\hline Bw1 & $11 / 13-22 / 26$ & $10 \mathrm{YR} 3 / 4$ & - & $1 \mathrm{p}$ & $\mathrm{gb}, \mathrm{hl}, \mathrm{sd}$ & $\mathrm{sg}$ & ba,bo \\
\hline Bw2 & $22 / 26-48$ & $10 \mathrm{YR} 4 / 4$ & - & $\operatorname{lp}$ & $\mathrm{gb}, \mathrm{hl}, \mathrm{sd}$ & $\mathrm{gb}$ & bu,r \\
\hline Bw3 & $48-78$ & $10 \mathrm{YR} 4 / 4$ & - & lpps & $\mathrm{gb}, \mathrm{hl}, \mathrm{sd}$ & $\mathrm{gb}$ & ba,r \\
\hline Bw4 & $78-100$ & $10 \mathrm{YR} 4 / 4$ & - & lpli & $\mathrm{gb}, \mathrm{hl}, \mathrm{sd}$ & $\mathrm{gb}$ & $\mathrm{ba}, \mathrm{r}$ \\
\hline $\mathrm{BC}$ & $100-150$ & $10 \mathrm{YR} 4 / 3$ & - & lp & $\mathrm{gb}, \mathrm{hl}, \mathrm{sd}$ & $\mathrm{gb}$ & ba,r \\
\hline \multicolumn{8}{|c|}{ Profiles 4/5 Humic Hapludults $25-40 \%$ mix garden } \\
\hline A & $0-10$ & $10 \mathrm{YR} 3 / 3$ & $10 \mathrm{YR} 5 / 4$ & lpli & $\mathrm{gb}, \mathrm{hl} \mathrm{sd}$ & $\mathrm{g}$ & ba,r \\
\hline $\mathrm{AB}$ & $10-28$ & $10 \mathrm{YR} 3 / 3$ & 10 YR 5/5 & lpli & $\mathrm{gb} \mathrm{hl} \mathrm{sd}$ & $\mathrm{g}$ & ba,r \\
\hline Bt1 & $28-44$ & $10 \mathrm{YR} 3 / 4$ & - & li & $\mathrm{gb} \mathrm{hl} \mathrm{ku}$ & $\mathrm{g}$ & bu,r \\
\hline $\mathrm{Bt} 2$ & $44-70$ & $10 \mathrm{YR} 3 / 4$ & - & li & $\mathrm{gb} \mathrm{hl} \mathrm{ku}$ & $\mathrm{g}$ & $\mathrm{bu}, \mathrm{r}$ \\
\hline Bt3 & $70-103$ & $10 \mathrm{YR} 4 / 4$ & - & li & $\mathrm{gb} \mathrm{hl} \mathrm{ku}$ & $\mathrm{g}$ & ba,r \\
\hline $\mathrm{BC}$ & $103-150$ & 10 YR 3.5/4 & - & li & $\mathrm{gb} \mathrm{hl} \mathrm{sd}$ & $\mathrm{g}$ & ba,r \\
\hline \multicolumn{8}{|c|}{ Profiles 5/6 Humic Hapludults $>40 \%$ mix garden } \\
\hline A & $0-12 / 14$ & $7.5 \mathrm{YR} 3 / 2$ & $7.5 \mathrm{YR} 5 / 5$ & Lpli & $\mathrm{gb}, \mathrm{hl}, \mathrm{sd}$ & $\mathrm{g}$ & ba,bo \\
\hline $\mathrm{AB}$ & $12 / 14-27$ & 7.5 YR 3/2 & 7.5 YR 5/4 & lpli & gb,hl,sd & $\mathrm{g}$ & $\mathrm{ba}, \mathrm{r}$ \\
\hline
\end{tabular}


The Classification and Characteristics of Soil Surrounding Lake Ecosystem as a Basic ......

\begin{tabular}{|c|c|c|c|c|c|c|c|}
\hline Bt1 & $27-41$ & 7.5 YR 4/4 & - & li & $\mathrm{gb}, \mathrm{hl}, \mathrm{ku}$ & $\mathrm{g}$ & ba,r \\
\hline Bt2 & $41-62$ & 7.5 YR 4/6 & - & Ipli & gb,hl,ku & $\mathrm{g}$ & bu,r \\
\hline Bt3 & $62-80$ & 7.5 YR 4/6 & - & li & gb,hl,ku & $\mathrm{g}$ & bu,r \\
\hline $\mathrm{Bt} 4$ & $80-100$ & $7.5 \mathrm{YR} 4 / 6$ & - & lpli & $\mathrm{gb}, \mathrm{hl}, \mathrm{ku}$ & $\mathrm{g}$ & bu,r \\
\hline Bt5 & $100-150$ & 7.5 YR 4/6 & - & lpli & $\mathrm{gb}, \mathrm{hl}, \mathrm{ku}$ & $\mathrm{g}$ & bu,r \\
\hline \multicolumn{8}{|c|}{ Profiles 9/7 Humic Hapludults $15-25 \%$ dryland } \\
\hline A & $0-9 / 10$ & $10 \mathrm{YR} \mathrm{3/3}$ & $10 \mathrm{YR} 4 / 5$ & lpli & gb,hl,sd & $\mathrm{gb}$ & ba,bo \\
\hline $\mathrm{Ab}$ & $9 / 10-30 / 31$ & $10 \mathrm{YR} 3 / 3$ & $10 \mathrm{YR} 4 / 5$ & lpli & $\mathrm{gb}, \mathrm{hl}, \mathrm{sd}$ & $\mathrm{gb}$ & ba,bo \\
\hline Bt1 & $30 / 31-53 / 56$ & $10 \mathrm{YR} 4 / 3$ & - & lide & $\mathrm{gb}, \mathrm{hl}, \mathrm{sd}$ & $\mathrm{gb}$ & ba,bo \\
\hline Bt2 & $53 / 56-91 / 93$ & $10 \mathrm{YR} 4 / 4$ & - & lide & $\mathrm{gb}, \mathrm{hl}, \mathrm{sd}$ & & ba,bo \\
\hline Bt3 & $91 / 93-150$ & $10 \mathrm{YR} 4 / 6$ & - & lide & gb,hl,sd & $\mathrm{gb}$ & ba,bo \\
\hline \multicolumn{8}{|c|}{ Profiles 2/8 Typic Sulfudepts $25-40 \%$ mix garden. sulphur } \\
\hline $\mathrm{A}$ & $0-23$ & $10 \mathrm{YR} 4 / 2$ & - & li & gb,hl,sd & $\mathrm{g}$ & ny,r \\
\hline Bw1 & $23-55$ & $10 \mathrm{YR} 4 / 2$ & - & li & gb,hl,sd & $\mathrm{g}$ & ba,r \\
\hline Bw2 & $55-93$ & $10 \mathrm{YR} 5 / 4$ & - & li & gb,hl,sd & $\mathrm{g}$ & ba,r \\
\hline $\mathrm{BC}$ & $93-150$ & 10 YR 8/12 & - & li & gb,hl,sd & $\mathrm{g}$ & $\mathrm{ba}, \mathrm{r}$ \\
\hline \multicolumn{8}{|c|}{ Profiles $8 / 9$, Sulfic Endoaquepts $>15 \%$, terrace, formerly use as rice field } \\
\hline Apg & $0-9$ & $\begin{array}{c}10 \mathrm{YR} 4 / 2-2.5 \mathrm{Y} \\
3 / 1 \\
\end{array}$ & - & li & gb,sd,sd, & $\mathrm{gb}$ & $\mathrm{j}, \mathrm{r}$ \\
\hline Bwg1 & $9-33$ & $\begin{array}{c}10 \mathrm{YR} 4 / 2-2.5 \mathrm{Y} \\
3 / 1 \\
\end{array}$ & - & li & $\mathrm{gb}, \mathrm{sd}, \mathrm{sd}$ & $\mathrm{gb}$ & ba,r \\
\hline Bwg2 & $33-56$ & $5 \mathrm{YR} \mathrm{3/4}$ & - & lide & $\mathrm{gb}, \mathrm{sd}, \mathrm{sd}$ & $\mathrm{gb}$ & ba,r \\
\hline Bwg3 & $56-85$ & $2.5 \mathrm{Y} \mathrm{3/1}$ & - & li & gb,hl,sd & $\mathrm{gb}$ & $\mathrm{j}, \mathrm{r}$ \\
\hline $\mathrm{BCg}$ & $85-100$ & $2.5 \mathrm{Y} 3 / 1$ & - & li & gb,hl,sd & $\mathrm{gb}$ & $\mathrm{j}, \mathrm{r}$ \\
\hline
\end{tabular}

\section{Remarks:}

Texture: sand (ps); silt (de); clay (li); loam (lp)

Structure: angular blocky (g), sub-angular Blocky (gb), fine (hl), medium, moderate (sd), strong (ku)

Consistency: friable (g), very friable $(\mathrm{sg})$

Boundary: undulating (bo); gradual (ba); diffuse (bu), abrupt (ny); clear (j), smooth (r)

Table 2. Some Soil Physical and Chemical Properties of Soil

\begin{tabular}{|c|c|c|c|c|c|c|c|c|c|c|c|c|c|c|c|c|c|}
\hline \multirow{2}{*}{$\begin{array}{c}\text { Hori- } \\
\text { zon }\end{array}$} & \multirow{2}{*}{$\begin{array}{c}\text { Sand } \\
(\%)\end{array}$} & \multirow{2}{*}{$\begin{array}{l}\text { Silt } \\
(\%)\end{array}$} & \multirow{2}{*}{$\begin{array}{l}\text { Clay } \\
(\%)\end{array}$} & \multirow{2}{*}{$\begin{array}{c}\text { BS } \\
(\% \\
) \\
\end{array}$} & \multirow{2}{*}{$\begin{array}{c}\mathrm{BD} \\
\mathrm{g} \\
\mathrm{cm}^{3}\end{array}$} & \multirow{2}{*}{$\begin{array}{c}\mathrm{pH} \\
\mathrm{H}_{2} \mathrm{O}\end{array}$} & \multirow{2}{*}{$\begin{array}{c}\text { Total } \\
\mathrm{N} \\
(\%)\end{array}$} & \multirow{2}{*}{$\begin{array}{l}\mathrm{C} / \mathrm{N} \\
\text { ratio }\end{array}$} & \multirow{2}{*}{$\begin{array}{c}\text { P. } \\
\text { Brayl } \\
\text { (mg.kg- } \\
\text { 1) }\end{array}$} & $\mathrm{K}$ & $\mathrm{Na}$ & $\mathrm{Ca}$ & $\mathrm{Mg}$ & CEC & $\begin{array}{l}\text { Exch } \\
\text {-Al }\end{array}$ & $\begin{array}{c}\text { Exch } \\
-H\end{array}$ & \multirow[t]{2}{*}{$\begin{array}{l}\mathrm{Fe} 203 \\
(\mathrm{ppm})\end{array}$} \\
\hline & & & & & & & & & & \multicolumn{7}{|c|}{$(\mathrm{NH} 40 \mathrm{AClNpH} 7)$} & \\
\hline \multicolumn{18}{|c|}{ P1 (LU 3) Profiles 1/3 Humic Dystrudepts $8-15 \%$ mix garden } \\
\hline A & 28 & 34 & 38 & 21 & & 4.4 & 0.28 & 9 & 5.76 & 0.34 & 0.16 & 7.92 & 0.51 & 41.94 & 10.66 & 1.64 & 932.36 \\
\hline Bw1 & 39 & 30 & 31 & 18 & 0.89 & 4.7 & 0.19 & 10 & 2.31 & 0.14 & 0.16 & 5.41 & 1.35 & 39.79 & 9.20 & 1.43 & 939.31 \\
\hline$B w 2$ & 42 & 31 & 27 & 18 & & 4.6 & 0.19 & 9 & 3.16 & 0.10 & 0.17 & 5.88 & 0.67 & 38.44 & 9.61 & 1.55 & 1005.89 \\
\hline Bw3 & 43 & 35 & 22 & 13 & & 4.1 & 0.04 & 26 & 0.58 & 0.01 & 0.13 & 5.13 & 0.34 & 42.55 & 18.17 & 2.45 & 825.80 \\
\hline $\mathrm{BC}$ & 37 & 35 & 28 & 12 & & 4.1 & 0.02 & 28 & 1.46 & 0.01 & 0.18 & 4.96 & 0.17 & 43.72 & 21.84 & 3.19 & 885.30 \\
\hline \multicolumn{18}{|c|}{ P5 (LU4) Profiles 6/4 Typic Dystrudepts, $15-25 \%$ mix garden } \\
\hline$A$ & 44 & 21 & 35 & 29 & & 5.3 & 0.26 & 9 & 7.45 & 0.22 & 0.38 & 9.06 & 1.65 & 38.80 & 1.86 & 0.69 & 1216.70 \\
\hline Bw1 & 43 & 32 & 25 & 25 & 0.91 & 5.1 & 0.15 & 9 & 3.14 & 0.08 & 0.40 & 7.02 & 3.01 & 41.63 & 1.34 & 0.68 & 1134.39 \\
\hline$B w 2$ & 38 & 36 & 26 & 30 & & 5.2 & 0.07 & 13 & 4.87 & 0.01 & 0.39 & 9.05 & 1.51 & 36.08 & 1.16 & 0.50 & 1207.64 \\
\hline Bw3 & 43 & 49 & 8 & 28 & & 5.0 & 0.04 & 17 & 6.62 & 0.01 & 0.40 & 8.59 & 1.68 & 38.55 & 0.99 & 0.32 & 1128.03 \\
\hline $\mathrm{Bw} 4$ & 37 & 34 & 29 & 29 & & 5.3 & 0.01 & 14 & 0.58 & 0.01 & 0.40 & 9.02 & 1.19 & 36.64 & 0.93 & 0.39 & 1094.83 \\
\hline $\mathrm{BC}$ & 38 & 42 & 19 & 30 & & 5.5 & 0.03 & 26 & 0.57 & 0.01 & 0.41 & 7.79 & 3.00 & 34.78 & 0.41 & 0.41 & 1382.32 \\
\hline \multicolumn{18}{|c|}{ P4/5 Profiles $4 / 5$ Humic Hapludults $25-40 \%$ mix garden } \\
\hline A & 26 & 37 & 37 & 11 & 0.69 & 5.0 & 0.29 & 10 & 3.96 & 0.45 & 0.34 & 2.98 & 0.36 & 37.88 & 2.10 & 0.91 & 1067.42 \\
\hline$A B$ & 22 & 52 & 26 & 25 & 0.98 & 5.5 & 0.16 & 10 & 1.50 & 0.27 & 0.29 & 6.49 & 1.50 & 34.73 & 1.16 & 0.50 & 1127.99 \\
\hline Bt1 & 19 & 35 & 46 & 26 & & 5.2 & 0.12 & 12 & 1.44 & 0.07 & 0.29 & 8.06 & 1.18 & 37.29 & 1.69 & 0.49 & 1027.50 \\
\hline $\mathrm{Bt} 2$ & 18 & 33 & 48 & 23 & & 5.3 & 0.10 & 10 & 1.44 & 0.01 & 0.30 & 6.25 & 1.18 & 38.79 & 2.62 & 1.35 & 965.00 \\
\hline $\mathrm{Bt} 3$ & 16 & 37 & 47 & 27 & & 4.9 & 0.10 & 11 & 1.45 & 0.02 & 0.30 & 8.48 & 1.53 & 38.79 & 2.16 & 0.74 & 1008.46 \\
\hline $\mathrm{BC}$ & 18 & 26 & 56 & 14 & & 4.9 & 0.07 & 12 & 1.44 & 0.01 & 0.30 & 6.25 & 1.18 & 38.79 & 2.62 & 1.35 & 1027,50 \\
\hline \multicolumn{18}{|c|}{ Profiles $5 / 6$ Humic Hapludults $>40 \%$ mix garden } \\
\hline A & 21 & 51 & 28 & 13 & 0.69 & 5.1 & 0.44 & 9 & 4.88 & 0.10 & 0.33 & 4.53 & 0.17 & 40.65 & 4.25 & 1.12 & 838.80 \\
\hline$A B$ & 23 & 46 & 31 & 20 & 0.98 & 5.4 & 0.25 & 11 & 2.30 & 0.01 & 0.33 & 5.39 & 1.68 & 37.39 & 1.34 & 0.54 & 773.88 \\
\hline $\mathrm{Bt1}$ & 18 & 31 & 50 & 21 & & 5.5 & 0.14 & 14 & 9.58 & 0.01 & 0.33 & 5.25 & 2.28 & \begin{tabular}{|l|l|}
37.72 \\
\end{tabular} & 2.21 & 0.61 & 1317.99 \\
\hline $\mathrm{Bt} 2$ & 28 & 39 & 39 & 16 & & 4.9 & 0.06 & 17 & 1.47 & 0.01 & 0.32 & 4.12 & 1.03 & \begin{tabular}{|l|}
33.51 \\
\end{tabular} & 18.06 & 10.36 & 1138.34 \\
\hline $\mathrm{Bt} 3$ & 21 & 39 & 40 & 13 & & 4.7 & 0.02 & 26 & 2.30 & 0.01 & 0.32 & 3.36 & 0.50 & 32.87 & 12.93 & 2.12 & 642.98 \\
\hline $\mathrm{Bt} 4$ & 24 & 30 & 36 & 10 & & 4.8 & 0.01 & 65 & 0.58 & 0.01 & 0.32 & 2.69 & 0.34 & 30.60 & 16.78 & 2.74 & 608.65 \\
\hline $\mathrm{Bt} 5$ & 26 & 46 & 28 & 10 & & 4.7 & 0.01 & 59 & 18.42 & 0.01 & 0.32 & 2.53 & 0.17 & 38.80 & 17.42 & 2.72 & 429.71 \\
\hline \multicolumn{18}{|c|}{ Profiles $9 / 7$ Humic Hapludults $15-25 \%$ dryland } \\
\hline A & 21 & 45 & 34 & 25 & & 5.5 & 0.33 & 9 & 1.42 & 0.06 & 0.30 & 6.83 & 0.50 & 31.24 & tu & 1.34 & 1484.51 \\
\hline $\mathrm{Ab}$ & 22 & 52 & 26 & 29 & 1.02 & 5.6 & 0.20 & 7 & 0.57 & 0.05 & 0.29 & 5.82 & 2.50 & 30.07 & to & 0.87 & 1370.03 \\
\hline
\end{tabular}


The Classification and Characteristics of Soil Surrounding Lake Ecosystem as a Basic .......

\begin{tabular}{|c|c|c|c|c|c|c|c|c|c|c|c|c|c|c|c|c|c|}
\hline $\mathrm{Bt1}$ & 26 & 43 & 31 & 25 & & 5.9 & 0.12 & 3 & 6.44 & 0.01 & 0.29 & 4.59 & 1.47 & 25.22 & to & 0.87 & 1713.62 \\
\hline $\mathrm{Bt} 2$ & 12 & 43 & 45 & 49 & & 5.5 & 0.09 & 7 & 1.46 & 0.01 & 0.30 & 7.17 & 1.54 & 18.28 & to & 0.64 & 117.360 \\
\hline $\mathrm{Bt} 3$ & 10 & 45 & 45 & 17 & & 5.2 & 0.07 & 4 & 0.58 & 0.05 & 0.32 & 7.60 & 2.53 & 63.27 & to & 0.52 & 1099.88 \\
\hline \multicolumn{18}{|c|}{ Profiles $2 / 8$ Typic Sulfudepts $25-40 \%$ mix garden. sulphur } \\
\hline A & 12 & 20 & 68 & 4 & & 4.0 & 0.26 & 12 & 29.20 & 0.10 & 0.22 & 1.54 & 0.17 & 56.33 & 34.95 & 5.74 & 1093.5 \\
\hline Bw1 & 13 & 20 & 67 & 4 & 1.11 & 4.0 & 0.18 & 14 & 15.18 & 0.04 & 0.23 & 1.71 & 0.17 & 51.74 & 35.07 & 5.58 & 1158.25 \\
\hline Bw2 & 17 & 25 & 58 & 5 & & 4.2 & 0.11 & 18 & 11.64 & 0.02 & 0.22 & 1.70 & 0.34 & 45.83 & 33.73 & 4.61 & 1069.09 \\
\hline $\mathrm{BC}$ & 19 & 26 & 55 & 4 & & 4.1 & 0.06 & 21 & 4.98 & 0.01 & 0.25 & 1.37 & 0.34 & 8.40 & 34.42 & 4.840 & 1055.54 \\
\hline \multicolumn{18}{|c|}{ Profiles $8 / 9$, Sulfic Endoaquepts $>15 \%$, terrace, formerly use as rice field } \\
\hline Apg & 18 & 40 & 42 & 26 & & 3.8 & 0.17 & 10 & 2.33 & 0.58 & 0.44 & 7.83 & 3.40 & 46.69 & 7.12 & 0.80 & 2267.87 \\
\hline Bwg1 & 15 & 42 & 43 & 31 & 1.08 & 4.0 & 0.15 & 9 & 2.30 & 0.55 & 0.41 & 7.91 & 4.54 & 43.91 & 5.47 & 3.67 & 2202.96 \\
\hline Bwg2 & 10 & 37 & 53 & 16 & & 3.7 & 0.15 & 9 & 2.32 & 0.09 & 0.41 & 2.55 & 0.68 & 57.94 & 13.20 & 7.24 & 2325.72 \\
\hline Bwg3 & 15 & 35 & 50 & 16 & & 3.0 & 0.05 & 10 & 4.05 & 0.08 & 0.33 & 4.74 & 0.85 & 38.53 & 4.50 & 14.49 & 2208.69 \\
\hline $\mathrm{BCg}$ & 11 & 47 & 42 & 14 & & 3.3 & 0.06 & 15 & 4.56 & 0.14 & 0.39 & 8.52 & 1.45 & 74.37 & 14.40 & 6.63 & 2583.37 \\
\hline
\end{tabular}

Remark: $\mathrm{BS}=$ base saturation, $\mathrm{CEC}=$ cation exchange capacity; Exch-Al = Exchangeable Aluminum; Exch-H $=$ Exchangeable Hidrogen. $\mathrm{tu}=$ trace

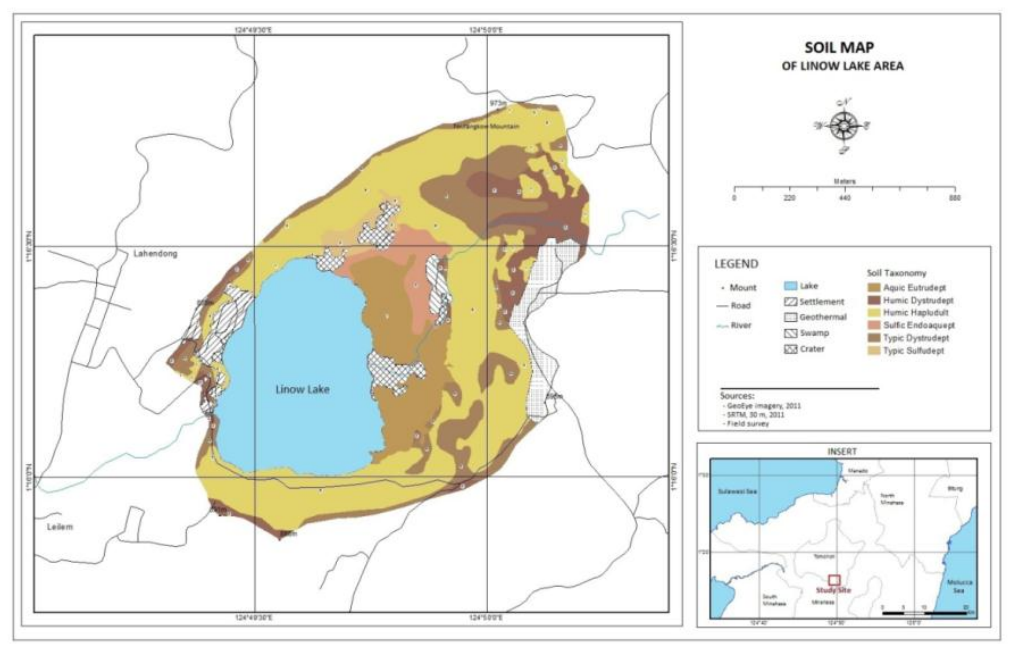

Fig. 3. The distribution of soil class in area adjacent to Linow Lake

\section{Land suitability evaluation for agrotourism development}

Lands capability and suitability are the important aspect in agrotourism development. It is become the fundamental aspect because agrotourism present the numerous productive agriculture products as tourism attraction. Fruit and vegetables are the main components of agrotourism product. Selecting the proper area for sustainable fruit trees and vegetables production therefore depends on the information of land capability and suitability. There are seven fruit trees that will be evaluated to be developed for the study area, namely Rambutan (Nephelium lappaceum), Lansat (Lansium domesticum), Avocado (Persea Americana), Durian (Durio zibethinus), Guava (Psidium guajava), Papaya (Carica papaya) and Banana (Musa paradisiacal).

As mention before the land use requirements and factor ratings, in this study was based on Djaenuddin et al. (2008) criteria [19]. There were thirteen lands characteristic of six land qualities that considered important in the area under study. According to climatic and soil data from Fig. 2 and Table 1 and 2, the land characteristic and land quality for each land unit or soil mapping unit (SMU) were shown on Table 3.

Table 3. The quality and characteristics of lands surrounding Linow Lake

\begin{tabular}{|l|l|l|l|l|l|l|l|l|}
\hline Land Quality & Land Characteristics & SMU 3 & SMU 4 & SMU 5 & SMU 6 & SMU 7 & SMU 8 & $\begin{array}{c}\text { SMU } \\
\mathbf{9}\end{array}$ \\
\hline \multirow{2}{*}{$\begin{array}{l}\text { Temperature, } \\
\text { tc }\end{array}$} & $\begin{array}{l}\text { - Average Temperature } \\
\left({ }^{\circ} \mathrm{C}\right)\end{array}$ & 22.7 & 22.7 & 22.7 & 22.7 & 22.7 & 22.7 & 22.7 \\
\cline { 2 - 9 } & - Elevation (m, asl) & 830 & 805 & 790 & 850 & 955 & 805 & 790 \\
\hline $\begin{array}{l}\text { Availability of } \\
\text { water, wa }\end{array}$ & - Rainfall (mm) & 259.41 & 259.41 & 259.41 & 259.41 & 259.41 & 259.41 & 259.41 \\
\cline { 2 - 9 } & Length of dry months & 3 & 3 & 3 & 3 & $3 \mathrm{~s}$ & 3 & 3 \\
\cline { 2 - 9 } & Average air humidity & 88 & 88 & 88 & 88 & 88 & 88 & 88 \\
\hline $\begin{array}{l}\text { Oxygen } \\
\text { availability, oa }\end{array}$ & -Soil Drainage & Well & Well & Well & Well & Well & Well & Well \\
\hline
\end{tabular}


The Classification and Characteristics of Soil Surrounding Lake Ecosystem as a Basic .......

\begin{tabular}{|l|l|l|l|l|l|l|l|l|}
\hline \multirow{2}{*}{$\begin{array}{l}\text { Rooting } \\
\text { Condition, rc }\end{array}$} & -Soil Texture & $\begin{array}{l}\text { Clay } \\
\text { loam }\end{array}$ & $\begin{array}{l}\text { Clay } \\
\text { loam }\end{array}$ & $\begin{array}{l}\text { Clay } \\
\text { loam }\end{array}$ & $\begin{array}{l}\text { Clay } \\
\text { loam }\end{array}$ & $\begin{array}{l}\text { Clay } \\
\text { loam }\end{array}$ & Clay & Clay \\
\cline { 2 - 9 } & -Soil depth (m) & 1.5 & 1.5 & 1.5 & 1.5 & 0.93 & 1.50 & 0.33 \\
\hline $\begin{array}{l}\text { Nutrient } \\
\text { Retention, nr }\end{array}$ & -CEC (meq/100 gr)) & 41.94 & 38.80 & 37.88 & 40.65 & 31.24 & 56.33 & 46.69 \\
\cline { 2 - 9 } & -Base saturation (\%) & 21 & 29 & 11 & 13 & 25 & 4 & 26 \\
\cline { 2 - 9 } & -pH H20 & 4.7 & 5.3 & 5.5 & 5.4 & 5.5 & 4.0 & 3.8 \\
\cline { 2 - 9 } & -Organic-C (\%) & 2.58 & 2.45 & 2.79 & 3.82 & 2.84 & 3.15 & 1.64 \\
\hline $\begin{array}{l}\text { Erosion } \\
\text { hazards (\%), eh }\end{array}$ & Slope (\%) & $\begin{array}{l}8 \\
\text { (mode- } \\
\text { rate) }\end{array}$ & $\begin{array}{l}15 \\
\text { (mode- } \\
\text { rate) }\end{array}$ & $\begin{array}{l}40 \\
\text { (high) }\end{array}$ & $\begin{array}{l}40 \\
\text { (high) }\end{array}$ & $\begin{array}{l}8 \text { (mode- } \\
\text { rate ) }\end{array}$ & $\begin{array}{l}25 \\
\text { (slightly } \\
\text { high) }\end{array}$ & $\begin{array}{l}15 \\
\text { (mode- } \\
\text { rate ) }\end{array}$ \\
\hline
\end{tabular}

By matching of land use requirements with land qualities using the Automated Land Evaluation Systems (ALES), the suitability of fruit trees for each map unit is shown on Table 4.

Based on Table 4, lands unit 5, 6 and 8 not suitable for fruit tree production. The erosion hazards due to the steep slopes, nutrients and water availability become the important aspect of such limitations. The slope of Land unit 5, 6 and 8 are ranging from 25 to $>40 \%$. In fact, however, some stands of Durian, Avocado and Lansium grows wild in such lands units.

Table 4. Soil types at study area.

\begin{tabular}{|l|l|l|c|c|}
\hline SMU & \multicolumn{1}{|c|}{ Landform } & \multicolumn{1}{c|}{ Soils } & Area (Ha) & Proportion from total area (\%) \\
\hline 1 & Lakustrin (swamp) & Aquic Eutrudept & 16.1 & 13 \\
\hline 2 & Hilly Volcanic & Humic Dystrudept & 3.8 & 6 \\
\hline 3 & Hilly Volcanic & Humic Dystrudept & 7.6 & 6 \\
\hline 4 & Hilly Volcanic & Typic Dystrudept & 23.4 & 18 \\
\hline 5 & Hilly Volcanic & Humic Hapludult & 33.2 & 26 \\
\hline 6 & Hilly Volcanic & Humic Hapludult & 28.7 & 23 \\
\hline 7 & Hilly Volcanic & Humic Hapludult & 5.4 & 2 \\
\hline 8 & Hilly Volcanic & Typic Sulfudept & 2.8 & 5 \\
\hline 9 & Hilly Volcanic & Sulfic Endoaquept & 5.7 & 100 \\
\hline \multicolumn{2}{|l}{} \\
\hline
\end{tabular}

Table 5. The actual land suitability of area surrounding Linow Lake for fruit trees cultivation

\begin{tabular}{|c|c|c|c|c|c|c|c|}
\hline \multirow{2}{*}{$\begin{array}{l}\text { Land unit } \\
\text { (SMU) }\end{array}$} & \multicolumn{7}{|c|}{ The actual land suitability of area surrounding Lake Linow } \\
\hline & $\begin{array}{l}\text { Rambutan } \\
\text { Nephelium } \\
\text { lappaceum }\end{array}$ & $\begin{array}{l}\text { Lansat Lansium } \\
\text { domesticum }\end{array}$ & $\begin{array}{l}\text { Avocado } \\
\text { Persea } \\
\text { americana } \\
\end{array}$ & $\begin{array}{l}\text { Durian Durio } \\
\text { zibethinus }\end{array}$ & $\begin{array}{l}\text { Guava } \\
\text { Psidium } \\
\text { guajava }\end{array}$ & $\begin{array}{l}\text { Papaya } \\
\text { Carica } \\
\text { papaya }\end{array}$ & $\begin{array}{l}\text { Banana } \\
\text { Musa } \\
\text { paradisiaca }\end{array}$ \\
\hline 1 & $\mathrm{~N}$ oa & Noa & Noa & Noa & Noa & Noa & Noa \\
\hline 2 & $\mathrm{~S} 2 \mathrm{wa} / \mathrm{nr}$ & S3nr & S3nr & S3nr & S3nr/wa & S3nr/wa & $\mathrm{S} 3 \mathrm{wa} / \mathrm{nr}$ \\
\hline 3 & S2wa & S3nr & S3nr & S3nr & S3nr/wa & S3nr/wa & S3wa \\
\hline 4 & S2eh/wa & S3eh & S3eh & S3eh & S3eh/wa & $\mathrm{S} 3 \mathrm{eh} / \mathrm{nr} / \mathrm{wa}$ & S3eh/wa \\
\hline 5 & Neh & Neh & Neh & Neh & Neh & Neh & Neh \\
\hline 6 & Neh & Neh & Neh & Neh & Neh & Neh & Neh \\
\hline 7 & S2eh/nr/ wa & S2eh/nr/wa & S2eh/nr/tc/wa & S2eh/nr/wa & S3wa & S3wa & S3wa \\
\hline 8 & S3eh/nr & $\mathrm{Neh} / \mathrm{nr}$ & $\mathrm{Neh} / \mathrm{nr}$ & $\mathrm{Neh} / \mathrm{nr}$ & $\mathrm{Neh} / \mathrm{nr}$ & $\mathrm{Neh} / \mathrm{nr}$ & $\mathrm{Neh} / \mathrm{nr}$ \\
\hline 9 & S3oa & S3eh/nr/oa & S3eh/nr/oa & S3eh/nr/oa & $\begin{array}{l}\text { S3eh/nr/oa/ } \\
\text { wa }\end{array}$ & $\begin{array}{l}\text { S3eh/nr/oa/ } \\
\text { wa }\end{array}$ & S3eh/oa/wa \\
\hline
\end{tabular}

\section{Implications for agrotourism development}

The agrotourism development, especially for its plants arrangement as tourism attractions, ultimately depend on the soil quality. Soils in area surrounding Linow Lake can be classified into two orders namely Inceptisols and Ultisols. Inceptisols is characterized by cambic horizon as shown on soil profile 1,2,6 and 7. Ultisols is characterized by the existence of argillic B horizon, as shown on soil profile 4,5 and 8 . The ultisol typically acid soil and commonly found in humid area. It is usually unsuitable for agriculture. In tropical countries with dominant ultisol soil, the richness of plants species in an area with dominant ultisol soil occurs as a result of dynamic equilibrium of nutrient cycling of ecosystems. The similar process seems to be found in Lands surrounding Linow Lake.

A phenomenon that many acid soils is ecologically covered by forest [25] lead the opportunities to introduce agroforestry techniques in lands surrounding Lake Linow. Agroforestry in the practices in which numerous plant trees species cultivated with crops to optimize economical and ecological benefits. Fruit trees is the important commodity among agroforestry practices in Indonesia. The opportunities for aroforestry based fruit trees in acid soil seem possible to implemented. In Thailand, numerous fruit threes has successfully cultivated in acid soils, such as Durio, Rambutan, Mangosteen, Longan, Litchi, and Mango [26]. These lead to 
opportunities for agrotourism in an area surrounding Linow Lake. Overall, the soil types and its percentages in study area for the development of agrotourism was given in Table 3.

Selecting the proper area for sustainable fruit and vegetables production therefore depends on the information of land capability and suitability. Based on Table 4, lands unit 5, 6 and 8 not suitable for fruit production. The erosion hazards due to land slopes, nutrients and water availability become the important aspect of such limitations. The land slope of Land unit 5, 6 and 8 are ranging from 25 to $>40 \%$. In fact, however, some stands of Durian, Avocado and Lansium grows wild in such lands units. Using plants against land erosion has been promoted in numerous countries [27]. Planting numerous trees in barren lands nowadays become one of the option of global restoration program, especially in tropical developing countries [28]. In order to enhance fruit plants productivities however, selecting plants which area suitable in particular area in Lake Linow is important. Land unit 2, 3, 4, 7, 8 and 9 were more suitable for almost all fruit trees that evaluated in this study. Rambutan has S2 suitability class for those land unit, while the other trees belongs to S3.

\section{Conclussion}

The area surrounding Linow Lake has opportunities to developed sustainably through agrotourism development. In such a acese, the information of soils classification and characteristics is important. Soils in adjacent to Linow Lake can be classified into two orders namely Inceptisols and Ultisols. These orders classified into five subgroup namely Humic Dystrudept, Typic Sulfudept, Humic Hapludult, Typic Dystrudept, Sulfic Endoaquept, and Aquic Eutrudept. The possibility for agrotourism development lies on the opportunities to plant some fruit trees, i.e. Rambutan, Lansat, Avocado, Durian, Guava, Papaya and Banana in policulture systems, or technically called agroforestry. The planting arragement and management should be meets the landcapability and suitability.

\section{Acknowledgements}

We wouldlike to thank Rector of Brawijaya University, Rector of Sam Ratulangi University, Director of Graduate Program Brawijaya Universty, Director of Gradute Program Sam Ratulangi University. The authors gratefully acknpowledege the assistance of Lab. of Soil Science, University of Brawijaya.

\section{References}

[1]. L. Hakim, Soemarno, SK. Hong, Challenges for conserving biodiversity and developing sustainable island tourism in North Sulawesi Province, Indonesia, Journal of Ecology and Field Biology, 35(2), 2012, 61-71.

[2]. A. Pangemanan, Maryunani, L. Hakim, B. Polii, Economic analysis of Bunaken National Park Ecotourism Area based on the carrying capacity and visitation level. Asian Transactions on Basic and Applied Sciences, 2(2), 2012, 34-40.

[3]. V.Justine, N. Bedford, M. Elliott, N. Ray, Indonesia (Lonely Planet Travel Guide, Lonely Planet, London, 2007)

[4]. GD. Cooke, EB. Welch, S. Peterson, SA. Nichols, Restoration and management of lakes and reservoirs (CRC press, 2005).

[5]. L. Hakim, C. Retnaningdyah, B. Yanuwiadi, Sunaryo, Project for Restoration of Ecosystem in Conservation Area in Bromo Tengger Semeru National Park, Phase I. Oktober 2010-Maret 2011 (Japan international Cooperation Agency, Jakarta, 2010).

[6]. MJ. Ntiba, WM. Kudoja, CT. Mukasa, Management issues in the Lake Victoria watershed. Lakes \& Reservoirs: Research \& Management, 6(3), 2001), 211-216.

[7]. JO. Ngana, RBB. Mwalyosi, NF. Madulu, PY. Yanda, Development of an integrated water resources management plan for the Lake Manyara sub-basin, Northern Tanzania. Physics and Chemistry of the Earth, Parts A/B/C, 28(20), 2003, $1033-1038$.

[8]. Landon. Tropical Soil: A Hand Book for Soil Survey and Agricultural land Evaluation in the tropics and subtropics (Booker Agricultural International Limited, 1984).

[9]. KJ. Coughland, NJ. McKenzie, H. Chresswell. Soil Physical Measurement and Interpretation For Land Evaluation (CSIRO Publishing Australia, 2002)

[10]. SW. Buol, FD. Hole, M. Cracken. Soil Genesis and Classification (Iowa State University Press, 2011).

[11]. BAPPEDA Kota Tomohon, Profil Kota Tomohon (Pemerintah Kota Tomohon, Sulawesi Utara, 2011).

[12]. Rayes L, Metode Inventarisasi Sumber Daya Lahan (ANDI Yogyakarta, 2006).

[13]. Soil Survey Staffs, Keys to Soil Taxonomy (United States Department of Agriculture. Washington, DC. 2010).

[14]. FAO, A Framework For Land Evaluation (Soil resources Management and Conservation Service Land and water Development Division, Italy, 1976).

[15]. GJ. Bouyoucos, A new calibration of the hydrometer methods for making mechanical analysis of soil, Agronomy Journal 43, 1951, 435-438.

[16]. JM. Bremner, Determination of Nitrogen in Soil by the Kjeldahl Method. The Journal of Agricultural Science, 55, $1960,11-33$.

[17]. LP. Van Reeuwijk, Procedures for soil Analysis. 6th ed. (International Soil References and Information Centre (SRIC), FAO-UN, 2002).

[18]. D.G. Rossiter, A.R. van Wambeke, Automated Land Evaluation System, ALES user Manual (Department of Soil, Crop \& Atmospheric Sciences (SCAS), Cornel University, Itacaha, 1997).

[19]. D. Djaenudin, Kriteria Kesesuaian lahan Untuk Komoditas Pertanian (Departemen Pertanian, Badan Penelitian dan Pengembangan Pertanian, 1997).

[20]. BMG, Informasi Tahunan Iklim Tomohon (BMG Kayuwatu, Tomohon, Manado, Sulawesi Utara, 2010).

[21]. MC. Peel, BL. Finlayson, TA. McMahon, "Updated world map of the Köppen-Geiger climate classification". Hydrol. Earth Syst. Sci. 11, 2007, 1633-1644.

[22]. J. Arnold, MR. Perez, Can non-timber forest products match tropical forest conservation and development objectives?. Ecological economics, 39(3), 2001, 437-447.

[23]. SW. Buol FD. Hole, McCracken, Soil Genesis and Classification (Iowa State University Press, 2011).

[24]. DS. Fanning, MCB. Fanning, Soil morphology, genesis and classification (John Wiley and Sons Inc., 1989). 
[25]. HR. Von Uexkiill, RP. Bosshart, Management of acid upland soils in Asia (Management of Acid Soils in the Humid Tropics of Asia, (2), 1989).

[26]. I. Kheoruenromne, P. Kesawapitakt, Management of Acid Soils for Food Crop Production in Thailand (Management of Acid Soils in the Humid Tropics of Asia, (100), 1989).

[27]. G.Gyssels, J. Poesen, E. Bochet, Y. Li, Impact of plant roots on the resistance of soils to erosion by water: a review. Progress in Physical Geography, 29(2), 2005, 189-217.

[28]. DL. Vieira, KD. Holl, FM. Peneireiro, Agro-Successional Restoration as a Strategy to Facilitate Tropical Forest Recovery. Restoration Ecology, 17(4), 2009, 451-459. 